\title{
Downregulation of TRAP1 aggravates injury of H9c2 cardiomyocytes in a hyperglycemic state
}

\author{
XIAODAN ZHANG ${ }^{*}, \mathrm{ZHEN} \mathrm{ZHONG}^{*}$ and WANGEN LI \\ Department of Endocrinology, The Second Affiliated Hospital of Guangzhou Medical University, \\ Guangzhou, Guangdong 510260, P.R. China
}

Received October 18, 2018; Accepted May 31, 2019

DOI: $10.3892 /$ etm.2019.7847

\begin{abstract}
Diabetic cardiomyopathy increases the risk of heart failure and is one of the major causes of death in patients with diabetes. The present study investigated the expression and function of tumor necrosis factor receptor-associated protein 1 (TRAP1) in cardiomyocytes in a hyperglycemic state. For the in vitro study, $\mathrm{H} 9 \mathrm{c} 2$ cells (rat cardiomyoblasts) were treated with normal glucose, high glucose. TRAP1 expression was determined by reverse transcription-quantitative PCR and western blot analysis. Viability of cardiomyocytes was detected using the CellTiter $96^{\circledR} \mathrm{AQ}_{\text {ueous }}$ One Solution assay. The intracellular reactive oxygen species (ROS) content was detected using a fluorescent 2',7'-dichlorodihydrofluorescein diacetate probe, and the change in mitochondrial membrane potential was detected by JC-1 fluorescent staining. Changes in cell viability, ROS content and mitochondrial membrane potential were determined following small interfering (si) RNA-mediated knockdown of TRAP1. Results demonstrated that compared with the normal control group, the expression of TRAP1 in H9c2 cells decreased in the high glucose group which was accompanied by a reduction in mitochondrial membrane potential and cell viability, and increased intracellular ROS production. TRAP1 expression was significantly decreased following TRAP1-siRNA transfection which was accompanied by enhanced ROS production, lower mitochondrial membrane potential and impaired cell viability. In conclusion, the present findings suggested that the decrease in cardiomyocyte TRAP1 expression under high glucose conditions was associated with myocardial injury. It was hypothesized that TRAP1 may have a protective role on cardiomyocytes under high glucose surroundings.
\end{abstract}

Correspondence to: Professor Wangen Li, Department of Endocrinology, The Second Affiliated Hospital of Guangzhou Medical University, 250 Changgang East Road, Guangzhou, Guangdong 510260, P.R. China

E-mail: liwg660@126.com

*Contributed equally

Key words: diabetic cardiomyopathy, tumor necrosis factor receptor-associated protein 1 , mitochondria, oxidative stress

\section{Introduction}

Diabetic cardiomyopathy (DCM), a heart disease that is independent of hypertension and coronary artery disease, mainly manifests as a cardiac dysfunction and a structural disorder. DCM increases the risk of heart failure and is one of the major causes of death in patients with diabetes (1). Pathogenesis of DCM is multifactorial, but hyperglycemia-induced overproduction of superoxides by the mitochondrial electron-transport chain is considered to be the common pathway leading to the condition (2). Mitochondria participate in the terminal part of the majority of apoptotic signaling pathways as 'gatekeepers' that ultimately determine cell survival and death (3). Mitochondrial dysfunction is closely related to multiple morbidities associated with DCM. A previous study demonstrated that compared with the control, the mass-specific respiration of streptozotocin-diabetic hearts was reduced during oxidative phosphorylation (4). Mitochondria in atrial tissues in individuals with type 2 diabetes demonstrated a markedly reduced capacity for glutamate and fatty acid-supported respiration compared with those of non-diabetic patients (5).

Tumor necrosis factor receptor-associated protein 1 (TRAP1) is a heat shock protein (HSP), which belongs to the HSP90 family, mainly located in mitochondria. TRAP1 is involved in a series of cell signaling pathways, participating in the regulation of mitochondrial function and cell oxidative stress (6). TRAP1 can protect cells and reduce apoptosis by decreasing the production of reactive oxygen species (ROS) and reducing oxidative stress (6). TRAP1 protects cancer cells from hypoxia-induced mitochondrial dysfunction and cell apoptosis during tumor progression, and is a key regulator of mitochondrial bioenergy that assists tumor cells in avoiding death and damage (7). Therefore, TRAP1 is a potential therapeutic target for designing novel anticancer agents (8). Previous studies determined that changes in TRAP1 expression in normal tissues also impact cellular function. TRAP1 serves a role in protecting mitochondria against damaging stimuli via the decrease of ROS generation (9). TRAP1 reduces cardiac hypertrophy and fibrosis that is induced by pressure overload through blocking the TAK/P38, JNK, and AKT signaling pathways (10). TRAP1 overexpression decreases ROS production and preserves mitochondrial membrane potential during glucose deprivation, while preserving ATP levels and cell viability during oxygen-glucose deprivation (11). However, the 
role of TRAP1 in DCM and its mechanisms of action remain undefined.

The present study aimed to investigate the role of TRAP1 in hyperglycemia-induced myocardial damage and to understand the associated mechanisms in cardiomyocytes under high glucose conditions.

\section{Materials and methods}

H9c2 cell culture. H9c2 cells (The Cell Bank of Type Collection of Chinese Academy of Sciences) were cultured with low-glucose DMEM medium (Gibco; Thermo Fisher Scientific, Inc.) containing 10\% FBS (Gibco; Thermo Fisher Scientific, Inc.), $1 \%$ penicillin-streptomycin solution (Gibco; Thermo Fisher Scientific, Inc.) and incubated with $5 \% \mathrm{CO}_{2}$ at $37^{\circ} \mathrm{C}$. When cell density reached $70-80 \%$ confluence, the cells were passaged in a 1:3 ratio, and logarithmic growth phase cells were used in subsequent experiments. The cells were grouped into normal glucose control group (C; $5.5 \mathrm{mmol} / 1$ glucose treatment for $48 \mathrm{~h}$; Sigma-Aldrich; Merck KGaA), high glucose group (G; $33 \mathrm{mmol} / 1$ glucose treatment for $48 \mathrm{~h}$ ), negative control small interfering RNA group (NC siRNA; negative control siRNA was transfected into $\mathrm{H} 9 \mathrm{c} 2$ cells for $24 \mathrm{~h}$, then cultured in the aforementioned hyperglycemic condition for $48 \mathrm{~h}$ ) and TRAP1 siRNA group (TRAP1 siRNA was transfected into H9c2 cells for $24 \mathrm{~h}$, then cultured in the aforementioned hyperglycemic conditions for $48 \mathrm{~h}$ ).

siRNA design and preparation. TRAP1 siRNA and NC siRNA sequences were designed and synthesized by GenePharma Co., Ltd. The sequences of the siRNAs were as follows: TRAP1 siRNA forward, 5'-GCUAGUGGAUCAGAUCUAUTT-3' and reverse, 5'-AUAGAUCUGAUCCACUAGCTT-3'; NC siRNA forward, 5'-UUCUCCGAACGUGUCACGUTT-3', and reverse: 5'-ACGUGACACGUUCGGAGAATT-3'. The siRNA products were vacuum centrifuged $(2,000 \mathrm{x}$ g at room temperature for $5 \mathrm{~min}$ ) and dissolved in diethyl pyrocarbonate-treated water (GenePharma Co., Ltd.) to obtain a $20 \mu \mathrm{M}$ stock solution before use.

siRNA transfection. H9c2 cells were seeded in six-well plates $\left(1.5 \times 10^{5}\right.$ cells/well) and cultured for $12 \mathrm{~h}$ to obtain fully adherent cells with a confluence of $30-50 \%$. Transfection was performed by adding $13 \mu \mathrm{l}$ of $20 \mu \mathrm{M}$ TRAP1 siRNA stock or NC siRNA stock and $6 \mu$ l Lipofectamine ${ }^{\circledR}$ RNAiMAX (Invitrogen; Thermo Fisher Scientific, Inc.) to $400 \mu 1$ Opti-MEM medium (Gibco; Thermo Fisher Scientific, Inc.). Following gentle mixing, the mixture was incubated at room temperature for $5 \mathrm{~min}$ to form a siRNA-transfection reagent mixture, which was then added to the six-well plate containing the cells, followed by addition of FBS-free DMEM to a final volume of $2 \mathrm{ml} /$ well. The medium was replaced with complete DMEM medium following $6 \mathrm{~h}$. After transfection with siRNA for $24 \mathrm{~h}$, the medium was replaced with high glucose DMEM for $48 \mathrm{~h}$ prior to subsequent experimentation.

Western blot analysis. Cells were washed twice with PBS, followed by addition of radioimmunoprecipitation assay lysis buffer (Beyotime Institute of Biotechnology) for $10 \mathrm{~min}$.
Next, the samples were kept on ice, sonicated $(130 \mathrm{~W}$; $\left.20 \mathrm{kHz} ; 30 \% ; 4^{\circ} \mathrm{C} ; 10 \mathrm{sec}\right)$, centrifuged $\left(13,680 \mathrm{x} \mathrm{g} ; 4^{\circ} \mathrm{C}\right.$; $5 \mathrm{~min}$ ) and the supernatant was added to the loading buffer (Hangzhou Fude Biological Technology Co., Ltd.) and boiled for $10 \mathrm{~min}$. Protein concentration was determined using a BCA assay. The proteins (30 $\mu \mathrm{g}$ per lane) were separated via SDS-PAGE on a $10 \%$ gel then transferred to polyvinylidene fluoride membranes. Membranes were blocked using 5\% non-fat dry milk at room temperature for $2 \mathrm{~h}$. Samples were incubated with primary antibodies anti-TRAP1 (1:1,000; cat. no. ab176478; Abcam) and GAPDH (1:3,000; cat. no. 5174; Cell Signaling Technology, Inc.) overnight at $4^{\circ} \mathrm{C}$. Following washes, membranes were incubated with horseradish peroxidase-conjugated goat anti-rabbit secondary antibody (1:5,000; cat. no. L153B; GeneCopoeia, Inc.) for $1 \mathrm{~h}$ at room temperature. This was followed by use of the FDbio-Femto Enhanced chemiluminescence kit (Hangzhou Fude Biological Technology Co., Ltd.) and exposure of the plate to a chemiluminescence imaging analyzer. Image $\mathbf{J}$ software (Image $\mathbf{J}$ 64-bit Java 1.8.0_112; National Institutes of Health) was used to analyze the gray-scale value of each band with GAPDH used as a loading control.

Reverse transcription-quantitative PCR (RT-qPCR). Total RNA was extracted from cells using TRIzolÒ (Thermo Fisher Scientific, Inc.). The quality and quantity of RNA samples were determined using Epoch ${ }^{\mathrm{TM}}$ microplate spectrophotometer (BioTek Instruments, Inc.). Total RNA was reverse transcribed into cDNA according to the instructions of PrimeScriptTM RT master mix (Takara Bio, Inc). qPCR was performed using Applied Biosystems ${ }^{\mathrm{TM}}$ PowerUp $^{\mathrm{TM}}$ SYBR $^{\mathrm{TM}}$ Green mix (Invitrogen; Thermo Fisher Scientific, Inc.) according to the manufacturer's instructions. The reaction volume total was $30 \mu 1$ and PCR was performed with LightCycler ${ }^{\circledR} 480$. the $2^{-\Delta \Delta \mathrm{Cq}}$ method was used for quantification (12). The primers used were as follows: TRAP1 forward, 5'-CTCAGTTGCTACAGCCCACA-3' and reverse, 5'-CTG CTATCATGGCGTTCTCA-3'; GAPDH forward, 5'-AGG TGAAGGTCGGAGTCAAC-3'; and reverse, 5'-CGCTCC TGGAAGATGGTGAT-3'.

MTS assay to determine cell viability. H9c2 cells were plated in 96-well plates at 5,000 cells/well. Viability was measured using the CellTiter $96^{\circledR} \mathrm{AQ}_{\text {ueous }}$ One Solution Assay kit (Promega Corporation). Culture medium was replaced with complete medium $(100 \mu \mathrm{l} / \mathrm{well})$ at the respective treatment time points. Then $20 \mu \mathrm{l}$ MTS assay reagent per well was added and incubated for $2 \mathrm{~h}$ at $37^{\circ} \mathrm{C}$. A microplate reader was used to detect the optical density (OD) at $490 \mathrm{~nm}$. The optical density is directly proportional to cell viability.

Intracellular ROS detection. ROS were detected using 2',7'-dichlorodihydrofluorescein diacetate (DCFH-DA) probe (Beyotime Institute of Biotechnology) according to the manufacturer's guidelines and fluorescence intensity was observed under a fluorescence microscope (magnification, $\mathrm{x} 40$ ). Fluorescence intensity was proportional to intracellular ROS levels. Image J software (Image J 64-bit Java 1.8.0_112; National Institutes of Health) was used to analyze the staining intensity of each group. 

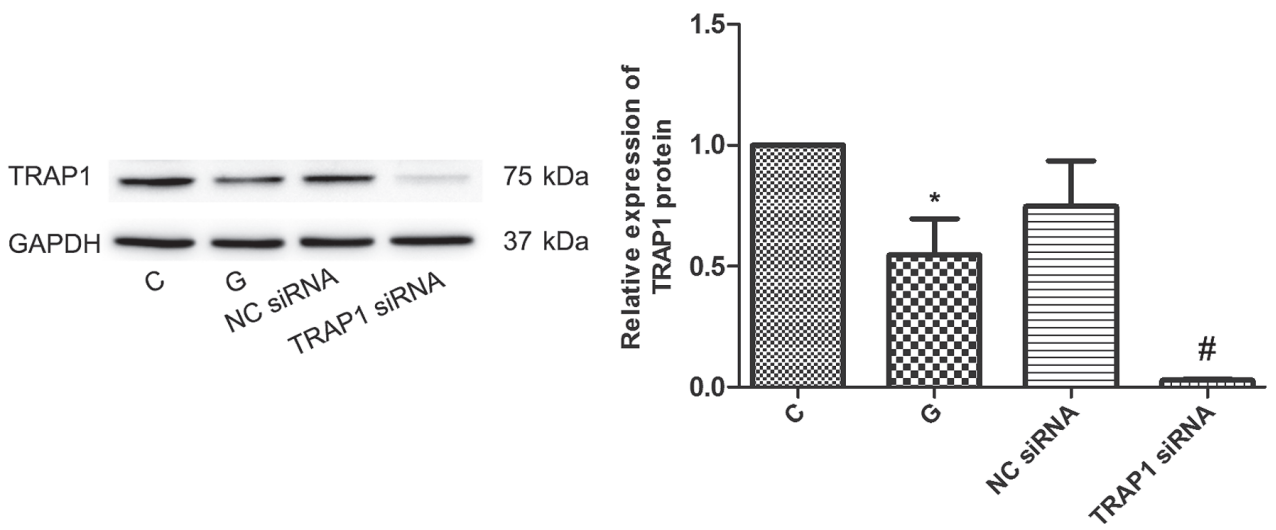

Figure 1. TRAP1 inhibition significantly decreases TRAP1 protein expression in H9c2 cells. Representative western blots and quantification following downregulation of TRAP1. " $\mathrm{P}<0.05$ vs. C group; ${ }^{*} \mathrm{P}<0.05$ vs. NC siRNA group. TRAP1, tumor necrosis factor receptor-associated protein 1 ; C, control group; $\mathrm{G}$, high glucose group; NC, negative control; siRNA, small interfering RNA.

Mitochondrial membrane potential. JC-1 fluorescence staining mitochondrial membrane potential detection kit (Beyotime Institute of Biotechnology) was used to detect changes in mitochondrial membrane potential according to the manufacturer's instructions. Fluorescence intensity was observed under a fluorescence microscope (magnification, 40x). Image J software (Image J 64-bit Java 1.8.0_112; National Institutes of Health) was used to analyze the staining intensity value of each group.

Statistical analysis. All data were analyzed using SPSS 16.0 statistical software (SPSS, Inc.) and expressed as the mean \pm standard deviation. This analysis was performed for three independent experiments at least. The differences between groups were analyzed using one-way analysis of variance followed by Least Significance Difference post hoc test. $\mathrm{P}<0.05$ was considered to indicate a statistically significant difference.

\section{Results}

TRAP1 inhibition significantly decreases TRAP1 protein and gene expression. siRNA-mediated knockdown efficiency was evaluated by detecting TRAP1 protein and mRNA levels by western blot analysis and RT-qPCR, respectively. Compared with $C$ group, the expression of TRAP1 protein was decreased significantly by $45 \%$ in $\mathrm{G}$ group $(\mathrm{P}<0.05$; Fig. 1$)$. Compared with the NC siRNA group, the expression of TRAP1 was significantly decreased by $96 \%$ in TRAP1 siRNA group ( $\mathrm{P}<0.05$; Fig. 1). Compared with $\mathrm{C}$ group, the level of TRAP1 mRNA was decreased significantly by $13 \%$ in $\mathrm{G}$ group (P<0.05; Fig. 2). Compared with NC siRNA group, the level of TRAP1 mRNA was significantly decreased by $87 \%$ in TRAP1 siRNA group ( $\mathrm{P}<0.05$; Fig. 2$)$.

Downregulation of TRAP1 reduces cell viability. Compared with the NC siRNA group, the downregulation of TRAP1 significantly reduced cell viability of $\mathrm{H} 9 \mathrm{c} 2$ cells $(\mathrm{P}<0.05$; Fig. 3). Relative cell viability values in the normal glucose control group, high glucose group, NC siRNA group and TRAP1 siRNA group were $1.02 \pm 0.07,0.83 \pm 0.06,0.83 \pm 0.07$ and $0.65 \pm 0.05$, respectively. The optical density is directly proportional to cell viability. The viability of $G$ group was

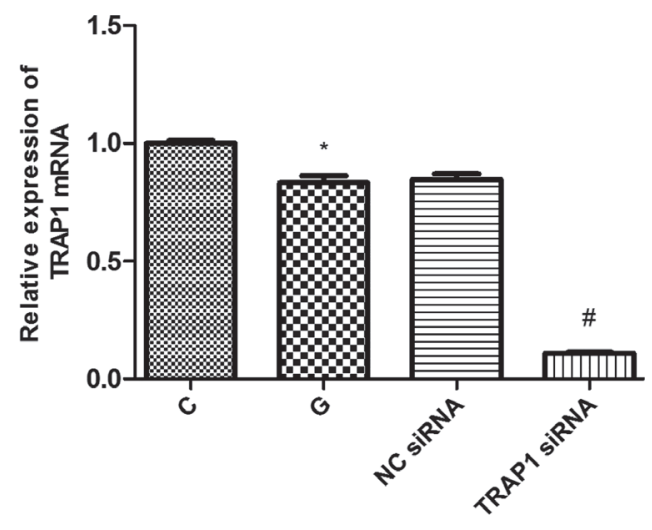

Figure 2.TRAP1 inhibition significantly decreases TRAP1 mRNA expression in $\mathrm{H} 9 \mathrm{c} 2$ cells. mRNA expression measured by reverse transcription-quantitative PCR following downregulation of TRAP1. ${ }^{*} \mathrm{P}<0.05$ vs. C group; ${ }^{\#} \mathrm{P}<0.05$ vs. NC siRNA group. TRAP1, tumor necrosis factor receptor-associated protein 1; C, control group; G, high glucose group; NC, negative control; siRNA, small interfering RNA.

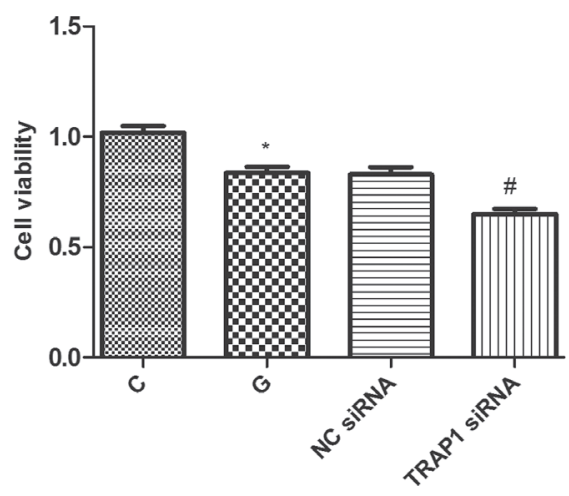

Figure 3. TRAP1 inhibition significantly decreases cell viability in $\mathrm{H} 9 \mathrm{c} 2$ cells. ${ }^{*} \mathrm{P}<0.05$ vs. $\mathrm{C}$ group; ${ }^{\#} \mathrm{P}<0.05$ vs. $\mathrm{NC}$ siRNA group. TRAP1, tumor necrosis factor receptor-associated protein 1; C, control group; G, high glucose group; NC, negative control; siRNA, small interfering RNA.

significantly lower compared with $\mathrm{C}$ group $(\mathrm{P}<0.05$; Fig. 3$)$. Downregulation of TRAP1 significantly reduced cell viability of $\mathrm{H} 9 \mathrm{c} 2$ cells compared with the NC siRNA group $(\mathrm{P}<0.05$; Fig. 3). 


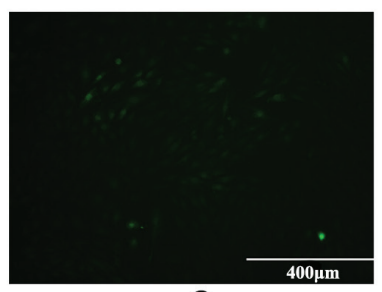

C

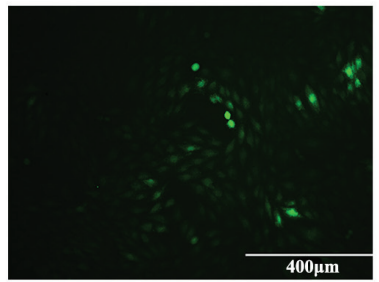

NC siRNA

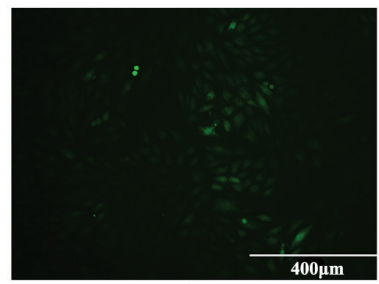

G

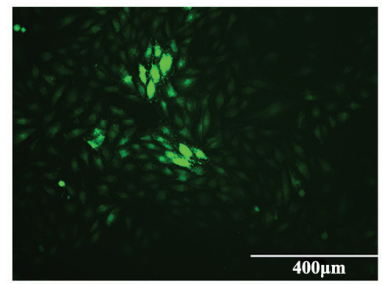

TRAP1 SiRNA

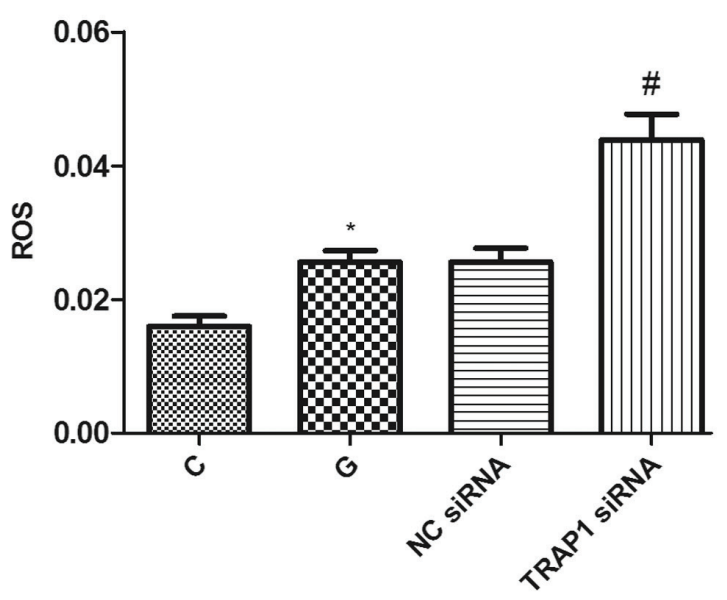

Figure 4. Downregulation of TRAP1 leads to ROS overproduction in H9c2 cells. Representative immunofluorescence micrographs demonstrating ROS content (green staining) and quantification of staining intensity. ${ }^{*} \mathrm{P}<0.05$ vs. normal control group; ${ }^{~} \mathrm{P}<0.05$ vs. NC siRNA group. TRAP1, tumor necrosis factor receptor-associated protein 1; ROS, reactive oxygen species; C, control group; G, high glucose group; NC, negative control; siRNA, small interfering RNA.

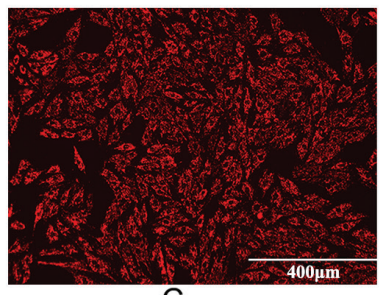

C

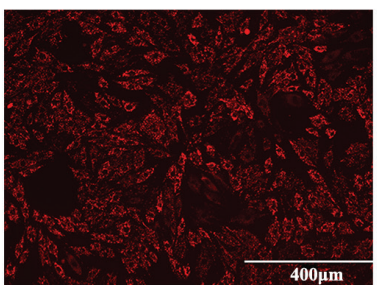

NC siRNA

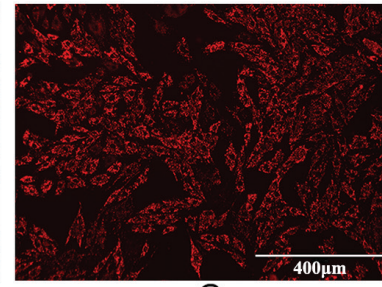

G

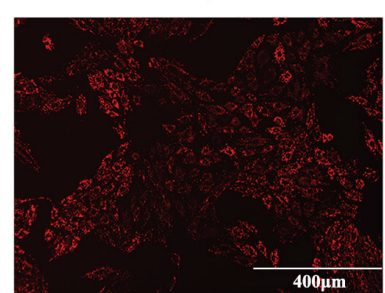

TRAP1 siRNA

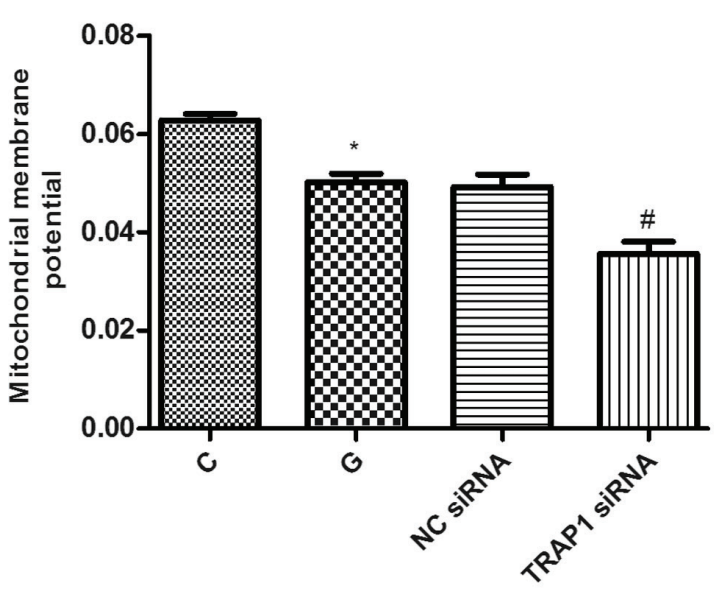

Figure 5. Downregulation of TRAP1 lowers the mitochondrial membrane potential in H9c2 cells. Representative immunofluorescence micrographs demonstrating the mitochondrial membrane potential (red staining) and quantification of staining intensity. ${ }^{*} \mathrm{P}<0.05$ vs. C group; ${ }^{\prime} \mathrm{P}<0.05 \mathrm{vs}$. NC siRNA group. TRAP1, tumor necrosis factor receptor-associated protein 1; C, control group; G, high glucose group; NC, negative control; siRNA, small interfering RNA.

Downregulation of TRAPl leads to ROS overproduction. The level of intracellular ROS can reflect the degree of oxidative stress in cells. Excessive ROS production can induce mitochondrial dysfunction of cardiomyocytes, thereby causing apoptosis (13). ROS content was investigated to determine whether cardiomyocyte injury was caused by the downregulation of TRAP1. The results demonstrated that the green fluorescence intensity of the $\mathrm{G}$ group was higher compared with $\mathrm{C}$ group $(\mathrm{P}<0.05$; Fig. 4$)$. The green fluorescence intensity is directly proportional to intracellular ROS content, and therefore, these results indicate that $\mathrm{H} 9 \mathrm{c} 2$ cells produced more ROS in the hyperglycemic state. Compared with NC siRNA group, the green fluorescence of TRAP1 siRNA group was significantly enhanced, suggesting that downregulation of TRAP1 in H9c2 cells led to ROS overproduction $(\mathrm{P}<0.05$; Fig. 4).

Downregulation of TRAP1 lowers the mitochondrial membrane potential. To investigate the effect of TRAP1 on mitochondrial function, the mitochondrial membrane potential in $\mathrm{H} 9 \mathrm{c} 2$ cells was investigated (Fig. 5). Results demonstrated that the red fluorescence intensity of $\mathrm{G}$ group was weaker compared with $\mathrm{C}$ group $(\mathrm{P}<0.05$; Fig. 5), suggesting that mitochondrial membrane potential of $\mathrm{H} 9 \mathrm{c} 2$ cells was reduced when cells were in the hyperglycemic state. Red fluorescence of cardiomyocytes transfected with TRAP1 siRNA was weaker $(\mathrm{P}<0.05$; Fig. 5) compared with NC siRNA group, indicating that downregulation of TRAP1 induced mitochondrial dysfunction in cardiomyocytes.

\section{Discussion}

DCM is one of the major causes of death in patients with diabetes (14). Insulin resistance, hyperinsulinemia and hyperglycemia are important factors triggering the development of DCM (15). The mechanisms involve multiple pathophysiological changes, including metabolic derangements, 
inappropriate activation of the renin-angiotensin-aldosterone system, oxidative stress, inflammation and immune dysfunction (16). Oxidative stress is considered to be an initiation factor for DCM development. Hyperglycemia and glucose fluctuation can induce acute oxidative stress, which leads to ROS accumulation, cellular dysfunction and cell death (17). The present study demonstrated that ROS levels increased and cardiomyocyte viability decreased when cells were in the hyperglycemic state, which was consistent with the literature $(18,19)$.

TRAP1 was first identified as a partner of tumor necrosis factor receptor 1 (TNFR1) in a yeast two-hybrid screen (20). Subsequently, it was demonstrated to be a molecular chaperone of the HSP90 family, as TRAP1 and HSP90 display $34 \%$ identity and $60 \%$ homology at the mRNA level and share the same domain organization (20). Previous studies demonstrated a pivotal role of TRAP1 in the pathogenesis of different tumors including breast, colon, pancreas, lung and nasopharyngeal adenocarcinoma with TRAP1 significantly upregulated in these malignancies (21-23). TRAP1 inhibition leads to impaired cell proliferation, mitochondrial lesion and reduced energy metabolism, and eventually results in apoptosis of tumor cells (24).

TRAP1 is variably expressed in multiple organs and tissues, including skeletal muscle, liver, heart, brain, kidney, pancreas, lung and placenta (20). It is mainly localized in the inner membrane of the mitochondria, and its primary function is to protect cells from mitochondrial apoptosis (6). TRAP1 is part of a supramolecular complex that is physically associated with HSP90, HSP60 and cyclophilin D (CypD) (25). CypD is a matrix peptidyl prolyl isomerase, which is a component of the organellar mitochondrial permeability transition pore (mPTP). This complex regulates mitochondrial function by controlling the mitochondrial permeability transition pore, cytochrome $\mathrm{C}$ release and the caspase cascade (26). TRAP1 can inhibit the opening of mPTP and reduce the release of cytochrome $\mathrm{C}$ and caspase 3 (27). Sorcin, a calcium binding protein of $18 \mathrm{kDa}$, is a TRAP1 client protein. Overexpression or downregulation of sorcin and/or TRAP1 demonstrated a reciprocal regulation between these two proteins and revealed that their interaction is required for sorcin mitochondrial localization and TRAP1 stability (28). Sorcin has a key role in controlling mitochondrial $\mathrm{Ca}^{2+}$ homeostasis, which assists in regulating the opening of the MPTP. TRAP1 chaperone activity may be crucial for sorcin folding, which contributes to mitochondrial $\mathrm{Ca}^{2+}$ homeostasis and is involved in $\mathrm{Ca}^{2+}$-dependent mPTP regulation (6). Silencing of TRAP1 via RNA interference increases ROS accumulation, whereas TRAP1 overexpression attenuates ROS production (29). Although TRAP1 is considered as 'mitochondrial HSP90', it also exists in extramitochondrial locations. TRAP1 interacts with the proteasomal particle TBP7 on the outer side of the endoplasmic reticulum (ER), and serves an important role in protein quality control. Inhibition of TRAP1 or TBP7 increases ER stress, apoptosis and intracellular protein ubiquitination $(30,31)$. Previous studies demonstrated that hypoxia induces an increase in TRAP1 expression in cardiomyocytes, and TRAP1 has a protective role by regulating the opening of the mPTP (32-34). TRAP1 provides cardioprotection against myocardial ischemia reperfusion by ameliorating mitochondrial dysfunction (35).

The present study demonstrated that TRAP1 levels were reduced in cardiomyocytes under hyperglycemic conditions, which was accompanied by increased ROS production, decreased mitochondrial membrane potential and impaired cell viability. Silencing of TRAP1 led to further deterioration of these changes, which indicated that TRAP1 may be involved in oxidative stress, mitochondrial dysfunction and cardiomyocyte lesion during hyperglycemia. However, the present study only involves preliminary in vitro research. Further in vitro and in vivo studies are required to investigate the role and underlying mechanisms of TRAP1 in DCM and to reinforce the current findings. Future work will involve establishment of a model of TRAP1 overexpression to investigate the changes of cardiomyocytes under high glucose surroundings.

In conclusion, the present study demonstrated that TRAP1 was associated with oxidative stress and mitochondrial dysfunction in cardiomyocytes cultured under hyperglycemic conditions. This suggested that TRAP1 may be involved in DCM development. To the best of our knowledge, this is the first study to investigate the expression and role of TRAP1 in cells that were in a high glucose state.

\section{Acknowledgements}

Not applicable.

\section{Funding}

The present study was supported by grants from the Natural Science Foundation of Guangdong, China (grant no. 2017A030310257), Science and Technology Program of Guangzhou, China (grant no. 201707010045) and the Doctoral Start-up Fund of Guangzhou Medical University (grant no. 2015C10).

\section{Availability of data and materials}

The datasets used and/or analyzed during the current study are available from the corresponding author on reasonable request.

\section{Authors' contributions}

$\mathrm{XZ}$ and WL designed experiments. ZZ and $\mathrm{XZ}$ performed experiments and analyzed data. $\mathrm{ZZ}$ drafted this manuscript and all authors approved this manuscript.

\section{Ethics approval and consent to participate}

Not applicable.

\section{Patient consent for publication}

Not applicable.

\section{Competing interests}

The authors declare that they have no competing interests. 


\section{References}

1. Dei Cas A, Fonarow GC, Gheorghiade M and Butler J: Concomitant diabetes mellitus and heart failure. Curr Probl Cardiol 40: 7-43, 2015.

2. Brownlee M: Biochemistry and molecular cell biology of diabetic complications. Nature 414: 813-820, 2001.

3. Granville DJ and Gottlieb RA: Mitochondria: Regulators of cell death and survival. ScientificWorldJournal 2: 1569-1578, 2002.

4. Pham T, Loiselle D, Power A and Hickey AJ: Mitochondrial inefficiencies and anoxic ATP hydrolysis capacities in diabetic rat heart. Am J Physiol Cell Physiol 307: C499-C507, 2014.

5. Anderson EJ, Kypson AP, Rodriguez E, Anderson CA, Lehr EJ and Neufer PD: Substrate-specific derangements in mitochondrial metabolism and redox balance in the atrium of the type 2 diabetic human heart. J Am Coll Cardiol 54: 1891-1898, 2009.

6. Matassa DS, Amoroso MR, Maddalena F, Landriscina M and Esposito F: New insights into TRAP1 pathway. Am J Cancer Res 2: 235-248, 2012

7. Masgras I, Sanchez-Martin C, Colombo G and Rasola A: The chaperone TRAP1 as a modulator of the mitochondrial adaptations in cancer cells. Front Oncol 7: 58, 2017.

8. Lettini G, Maddalena F, Sisinni L, Condelli V, Matassa DS, Costi MP, Simoni D, Esposito F and Landriscina M: TRAP1: A viable therapeutic target for future cancer treatments? Expert Opin Ther Targets 21: 805-815, 2017.

9. Im CN, Lee JS, Zheng Y and Seo JS: Iron chelation study in a normal human hepatocyte cell line suggests that tumor necrosis factor receptor-associated protein 1 (TRAP1) regulates production of reactive oxygen species. J Cell Biochem 100: 474-486, 2007.

10. Zhang Y, Jiang DS, Yan L, Cheng KJ, Bian ZY and Lin GS: HSP75 protects against cardiac hypertrophy and fibrosis. J Cell Biochem 112: 1787-1794, 2011.

11. Voloboueva LA, Duan M, Ouyang Y, Emery JF, Stoy C and Giffard RG: Overexpression of mitochondrial Hsp70/Hsp75 protects astrocytes against ischemic injury in vitro. J Cereb Blood Flow Metab 28: 1009-1016, 2008.

12. Livak KJ and Schmittgen TD: Analysis of relative gene expression data using real-time quantitative PCR and the 2(-Delta Delta C(T)) method. Methods 25: 402-408, 2001.

13. Roul D and Recchia FA: Metabolic alterations induce oxidative stress in diabetic and failing hearts: Different pathways, same outcome. Antioxid Redox Signal 22: 1502-1514, 2015.

14. Bragg F, Holmes MV, Iona A, Guo Y, Du H, Chen Y, Bian Z, Yang L, Herrington W, Bennett D, et al: Association between diabetes and cause-specific mortality in rural and urban areas of China. JAMA 317: 280-289, 2017.

15. Lee WS and Kim J: Diabetic cardiomyopathy: Where we are and where we are going. Korean J Intern Med 32: 404-421, 2017.

16. Jia G, Whaley-Connell A and Sowers JR: Diabetic cardiomyopathy: A hyperglycaemia- and insulin-resistance-induced heart disease. Diabetologia 61: 21-28, 2018.

17. Liu ZW, Zhu HT, Chen KL, Dong X, Wei J, Qiu C and Xue JH: Protein kinase RNA-like endoplasmic reticulum kinase (PERK) signaling pathway plays a major role in reactive oxygen species (ROS)-mediated endoplasmic reticulum stress-induced apoptosis in diabetic cardiomyopathy. Cardiovasc Diabetol 12: 158, 2013.

18. Huynh K, Bernardo BC, Mcmullen JR and Ritchie RH: Diabetic cardiomyopathy: Mechanisms and new treatment strategies targeting antioxidant signaling pathways. Pharmacol Ther 142: 375-415, 2014

19. Liu Q, Wang S and Cai L: Diabetic cardiomyopathy and its mechanisms: Role of oxidative stress and damage. J Diabetes Investig 5: 623-634, 2014

20. Song HY, Dunbar JD, Zhang YX, Guo D and Donner DB: Identification of a protein with homology to hsp90 that binds the type 1 tumor necrosis factor receptor. J Biol Chem 270: 3574-3581, 1995
21. Zhang B, Wang J, Huang Z, Wei P, Liu Y, Hao J, Zhao L, Zhang F, $\mathrm{Tu} \mathrm{Y}$ and Wei T: Aberrantly upregulated TRAP1 is required for tumorigenesis of breast cancer. Oncotarget 6: 44495-44508, 2015.

22. Gao JY, Song BR, Peng JJ and Lu YM: Correlation between mitochondrial TRAP-1 expression and lymph node metastasis in colorectal cancer. World J Gastroenterol 18: 5965-5971, 2012.

23. Lee JH, Kang KW, Kim JE, Hwang SW, Park JH, Kim SH, Ji JH, Kim TG, Nam HY, Roh MS, et al: Differential expression of heat shock protein 90 isoforms in small cell lung cancer. Int J Clin Exp Pathol 8: 9487-9493, 2015.

24. Yuan M and Li X: Research progress of the relationship between TRAP1 and malignant tumor. Shi Yong Zhong Liu Xue Za Zhi 31: 371-375, 2017 (In Chinese)

25. Montesano Gesualdi N, Chirico G, Pirozzi G, Costantino E, Landriscina $\mathrm{M}$ and Esposito F: Tumor necrosis factor-associated protein 1 (TRAP-1) protects cells from oxidative stress and apoptosis. Stress 10: 342-350, 2007.

26. Kang BH, Plescia J, Dohi T, Rosa J, Doxsey SJ and Altieri DC: Regulation of tumor cell mitochondrial homeostasis by an organelle-specific Hsp90 chaperone network. Cell 131: 257-270, 2007.

27. Altieri DC, Stein GS, Lian JB and Languino LR: TRAP-1, the mitochondrial Hsp90. Biochim Biophys Acta 1823: 767-773, 2012.

28. Landriscina M, Laudiero G, Maddalena F, Amoroso MR, Piscazzi A, Cozzolino F, Monti M, Garbi C, Fersini A, Pucci P and Esposito F: Mitochondrial chaperone Trap1 and the calcium binding protein Sorcin interact and protect cells against apoptosis induced by antiblastic agents. Cancer Res 70: 6577-6586, 2010.

29. Hua G, Zhang Q and Fan Z: Heat shock protein 75 (TRAP1) antagonizes reactive oxygen species generation and protects cells from granzyme M-mediated apoptosis. J Biol Chem 282: 20553-20560, 2007.

30. Amoroso MR, Matassa DS, Laudiero G, Egorova AV, Polishchuk RS, Maddalena F, Piscazzi A, Paladino S, Sarnataro D, Garbi C, et al: TRAP1 and the proteasome regulatory particle TBP7/Rpt3 interact in the endoplasmic reticulum and control cellular ubiquitination of specific mitochondrial proteins. Cell Death Differ 19: 592-604, 2012.

31. Amoroso MR, Matassa DS, Sisinni L, Lettini G, Landriscina M and Esposito F: TRAP1 revisited: Novel localizations and functions of a 'next-generation' biomarker (review). Int J Oncol 45: 969-977, 2014.

32. Xiang F, Huang YS, Shi XH and Zhang Q: Mitochondrial chaperone tumour necrosis factor receptor-associated protein 1 protects cardiomyocytes from hypoxic injury by regulating mitochondrial permeability transition pore opening. FEBS J 277: 1929-1938, 2010.

33. Xiang F, Ma SY, Lv YL, Zhang DX, Song HP and Huang YS: Tumor necrosis factor receptor-associated protein 1 regulates hypoxia-induced apoptosis through a mitochondria-dependent pathway mediated by cytochrome c oxidase subunit II. Burns Trauma 7: 16, 2019.

34. Xiang F, Zhang DX, Ma SY and Huang YS: Mechanism of protective effects of tumor necrosis factor receptor associated protein 1 on hypoxic cardiomyocytes of rats. Zhonghua Shao Shang Za Zhi 32: 744-751, 2016 (In Chinese).

35. Zhang P, Lu Y, Yu D, Zhang D and Hu W: TRAP1 provides protection against myocardial ischemia-reperfusion injury by ameliorating mitochondrial dysfunction. Cell Physiol Biochem 36: 2072-2082, 2015. 